\title{
SOME SUFFICIENT CONDITIONS FOR A DETERMINATE HAMBURGER MOMENT SEQUENCE ${ }^{1}$
}

FRED M. WRIGHT

1. Introduction. Throughout this paper, let

$$
\left\{\mu_{n}\right\}, \quad(n=0,1,2, \cdots)
$$

be a given positive definite Hamburger moment sequence. Let

$$
\frac{a_{0}^{2}}{z+b_{1}}-\frac{a_{1}^{2}}{z+b_{2}}-\frac{a_{2}^{2}}{z+b_{3}}-\cdots
$$

be the $J$-fraction expansion of the formal power series $\sum_{n=0}^{\infty} \mu_{n} / z^{n+1}$. From a result of Stieltjes $[1]^{2}$ we have that

$$
\mu_{p+q}=(-1)^{p+q} \cdot \sum_{i=0}^{\infty} a_{0}^{2} a_{1}^{2} a_{2}^{2} \cdots a_{i}^{2} k_{i, p} k_{i, q} \quad(p, q=0,1,2, \cdots),
$$

where the constants $k_{p, q}$ are given by

$$
\begin{aligned}
& k_{0,0}=1, \quad k_{p, q}=0 \text { if } p>q,
\end{aligned}
$$

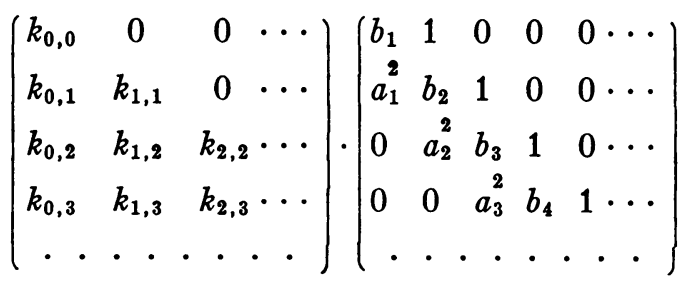

$$
\begin{aligned}
& =\left(\begin{array}{ccccc}
k_{0,1} & k_{1,1} & 0 & 0 & 0 \\
k_{0,2} & k_{1,2} & k_{2,2} & 0 & 0 \\
k_{0,3} & k_{1,3} & k_{2,3} & k_{3,3} & 0 \\
. & \ldots & \ldots & \ldots & \ldots \\
. & \ldots & \ldots
\end{array}\right) .
\end{aligned}
$$

In this paper, we shall make use of

LEMMA 1.1. (1.1) is a determinate Hamburger moment sequence in case $\sum_{n=0}^{\infty} 1 /\left|a_{n}\right|=+\infty[2 ; 3]$.

Presented to the Society, April 22, 1955; received by the editors September 1, 1955 and, in revised form, November 17, 1955.

${ }^{1}$ The material in this paper is contained in a thesis submitted to Northwestern University and prepared under the direction of Professor Walter T. Scott.

${ }^{2}$ Numbers in brackets refer to references at the end of the paper. 
We shall also use

Lemma 1.2 (CARLeman's inequality). If $\left\{u_{n}\right\}(n=1,2,3, \cdots)$, is an infinite sequence of non-negative real numbers such that $u_{1}>0$, then [4],

$$
\sum_{p=1}^{n}\left(u_{1} u_{2} \cdots u_{p}\right)^{1 / p}<e \cdot \sum_{r=1}^{n} u_{r} \quad(n=1,2,3, \cdots) .
$$

In $\$ 2$ we obtain, with the aid of formulas (1.3) as well as Lemma 1.1 and Lemma 1.2, a relatively simple infinite sequence of sufficient conditions for (1.1) to be a determinate Hamburger moment sequence. It is shown that the famous Carleman criterion for a determinate Hamburger moment sequence is weaker than any of these conditions. It is to be noted that there are infinitely many more relationships than those used in this paper available from (1.4) for one to use in developing sufficient conditions for (1.1) to be a determinate Hamburger moment sequence, although these relationships may prove difficult to manipulate and may yield complicated results. Moreover, most of the conditions developed in $\$ 2$ can be strengthened, but the stronger conditions are more complicated.

2. An infinite sequence of sufficient conditions. If $\boldsymbol{n}$ is any nonnegative integer and $j$ is any positive integer, then

$$
\mu_{2 n+j}=(-1)^{i} \cdot \sum_{i=0}^{n} a_{0}^{2} a_{1}^{2} a_{2}^{2} \cdots a_{i}^{2} k_{i, n} k_{i, n+j}
$$

in view of (1.3) and hence

$$
\begin{aligned}
{\left[\mu_{2 n+i}\right]^{2} } & =\left[\sum_{i=0}^{n}\left(a_{0} a_{1} a_{2} \cdots a_{i} k_{i, n}\right) \cdot\left(a_{0} a_{1} a_{2} \cdots a_{i} k_{i, n+j}\right)\right]^{2} \\
& \leqq \sum_{i=0}^{n}\left(a_{0}^{2} a_{1}^{2} a_{2}^{2} \cdots a_{i}^{2} k_{i, n}^{2}\right) \cdot \sum_{i=0}^{n}\left(a_{0}^{2} a_{1}^{2} a_{2}^{2} \cdots a_{i}^{2} k_{i, n+j}^{2}\right)
\end{aligned}
$$

in view of Schwarz's inequality. Therefore, if $n$ is any non-negative integer and $j$ is any positive integer, we have, in view of (1.3), that

$$
\left[\mu_{2 n+i}\right]^{2} \leqq \mu_{2 n} \cdot\left[\mu_{2 n+2 j}-\sum_{i=n+1}^{n+j}\left(a_{0}^{2} a_{1}^{2} a_{2}^{2} \cdots a_{i}^{2} k_{i, n+j}^{2}\right)\right] .
$$

Since $k_{p, p}=1$ for each integer $p=0,1,2, \cdots$ in view of $(1.4)$, we then have that

$$
\begin{aligned}
{\left[\mu_{2 n} \mu_{2 n+2 j}-\underset{\mu_{2 n+j}}{2}\right] } & \geqq \underset{a_{0} a_{1}^{2} a_{2}^{2} \cdots a_{n+j}^{2} \cdot \mu_{2 n},}{ } \\
& (j=1,2,3, \cdots ; n=0,1,2, \cdots) .
\end{aligned}
$$


From (2.2) it follows that

(2.3) $\left[\mu_{2 n} \mu_{2 n+2 j}-\mu_{2 n+i}^{2}\right]>0, \quad(j=1,2,3, \cdots ; n=0,1,2, \cdots)$.

From (2.1) it follows that

$$
\begin{aligned}
& 0<\left(\frac{\mu_{2 n}}{\mu_{2 n} \mu_{2 n+2 j}-\mu_{2 n+j}^{2}}\right)^{1 /(2 n+2 j)} \\
& \leqq\left(\frac{1}{\left|a_{0} a_{1}\right| \cdot\left|a_{2}\right| \cdot\left|a_{3}\right| \cdots\left|a_{n+j}\right|}\right)^{1 /(n+j)}, \\
& \quad(j=1,2,3, \cdots ; n=0,1,2, \cdots) .
\end{aligned}
$$

We note then that

$$
\begin{aligned}
& \sum_{n=0}^{m}\left(\frac{1}{\left|a_{0} a_{1}\right| \cdot\left|a_{2}\right| \cdot\left|a_{3}\right| \cdots\left|a_{n+j}\right|}\right)^{1 /(n+j)} \\
& \quad=\sum_{p=j}^{j+m}\left(\frac{1}{\left|a_{0} a_{1}\right| \cdot\left|a_{2}\right| \cdot\left|a_{3}\right| \cdots\left|a_{p}\right|}\right)^{1 / p} \\
& \leqq \sum_{p=1}^{i+m}\left(\frac{1}{\left|a_{0} a_{1}\right| \cdot\left|a_{2}\right| \cdot\left|a_{3}\right| \cdots\left|a_{p}\right|}\right)^{1 / p} \\
&<e \cdot\left(\frac{1}{\left|a_{0} a_{1}\right|}+\sum_{r=2}^{j+m} \frac{1}{\left|a_{r}\right|}\right), \quad(m, j=1,2,3, \cdots)
\end{aligned}
$$

in view of Lemma 1.2. From (2.4) and Lemma 1.1 we then have the following denumerable set of rather simple sufficient conditions for (1.1) to be a determinate Hamburger moment sequence.

TheOREM 2.1. (1.1) is a determinate Hamburger moment sequence in case at least one of the conditions

$$
\sum_{n=0}^{\infty}\left(\frac{\mu_{2 n}}{\mu_{2 n} \mu_{2 n+2 j}-\mu_{2 n+j}^{2}}\right)^{1 /(2 n+2 j)}=+\infty, \quad(j=1,2,3, \cdots)
$$

holds.

We note that

$$
\frac{1}{\mu_{2 n+2 j}} \leqq \frac{\mu_{2 n}}{\mu_{2 n} \mu_{2 n+2 j}-\mu_{2 n+j}^{2}},(j=1,2,3, \cdots ; n=0,1,2, \cdots) .
$$

From Theorem 2.1 and (2.6) we have the following famous sufficient condition, known as Carleman's criterion, for (1.1) to be a determinate Hamburger moment sequence; although this condition is 
evidently weaker than any of the sufficient conditions of Theorem 2.1 , it is quite "useful" and well known because of its simplicity.

THEOREM 2.2. (1.1) is a determinate Hamburger moment sequence in case $[4 ; 5 ; 6]$

$$
\sum_{n=1}^{\infty}\left(\frac{1}{\mu_{2 n}}\right)^{1 / 2 n}=+\infty .
$$

It is clear that stronger sufficient conditions than those of Theorem 2.1 can be obtained by using sharper inequalities than (2.2) and more of the relations (1.4) than those used here. The author has obtained an infinite sequence of sufficient conditions, each stronger than the corresponding condition of Theorem 2.1 for $j>1$, by retaining one more term on the right side of (2.1) to form sharper inequalities (2.2). Each condition of this sequence is somewhat more complicated than the corresponding condition of Theorem 2.1, but these conditions are still fairly simple. However, a detailed discussion of these conditions will be omitted here in the interest of brevity.

\section{REFERENCES}

1. T. J. Stieltjes, Sur la réduction en fraction continue d'une série précédent suivant les pouissances descendants d'une variable, Ann. Fac. Sci. Univ. Toulouse vol. 3 (1889) pp. 1-17.

2. J. J. Dennis and H.S. Wall, The limit-circle case for a positive definite $J$-fraction, Duke Math. J. vol. 12 (1955) pp. 255-273.

3. R. Nevalinna, Asymptotische Entwicklungen beschränkter Funktionen und das Stieltjesschen Momentenproblem, Annales Academiae Fennicae (A) vol. 18 (1922) no. 5.

4. T. Carleman, Les fonctions quasi analytiques, Paris, 1926, pp. 78-96.

5. - Sur les équations integrales singulières à noyau symétrique, Uppsala, 1923, pp. 189-220.

6. H. S. Wall, Analytic theory of continued fractions, Van Nostrand, 1948.

NORTHWESTERN UNIVERSITY 